Supporting Information (SI)

\title{
All-inorganic Perovskite Nanorod Arrays with Spatially Randomly Distributed Lasing Modes for All-photonic Cryptographic Primitives
}

Xinlian Chen ${ }^{a, \#, ~ K a i y a n g ~ W a n g, ~}{ }^{b, \#, ~ B o r i ~ S h i a, \#, ~ T a n g h a o ~ L i u ~}{ }^{b}$, Riming Chen ${ }^{b}$, Mengying Zhang ${ }^{c^{*}}$, Weijia Wen ${ }^{a, d}$, Guichuan Xing ${ }^{b}$ and Jinbo Wu $u^{a^{*}}$

a Materials Genome Institute, Shanghai University, Shanghai 200444, China.

b Joint Key Laboratory of the Ministry of Education, Institute of Applied Physics and Materials Engineering, University of Macau, Avenida da Universidade, Taipa, Macau 999078, China

c Department of Physics, Shanghai University, Shanghai 200444, China.

d Department of Physics, The Hong Kong University of Science and Technology, Hong Kong, China.

*Email: jinbowu@t.shu.edu.cn; zhang.my@t.shu.edu.cn 


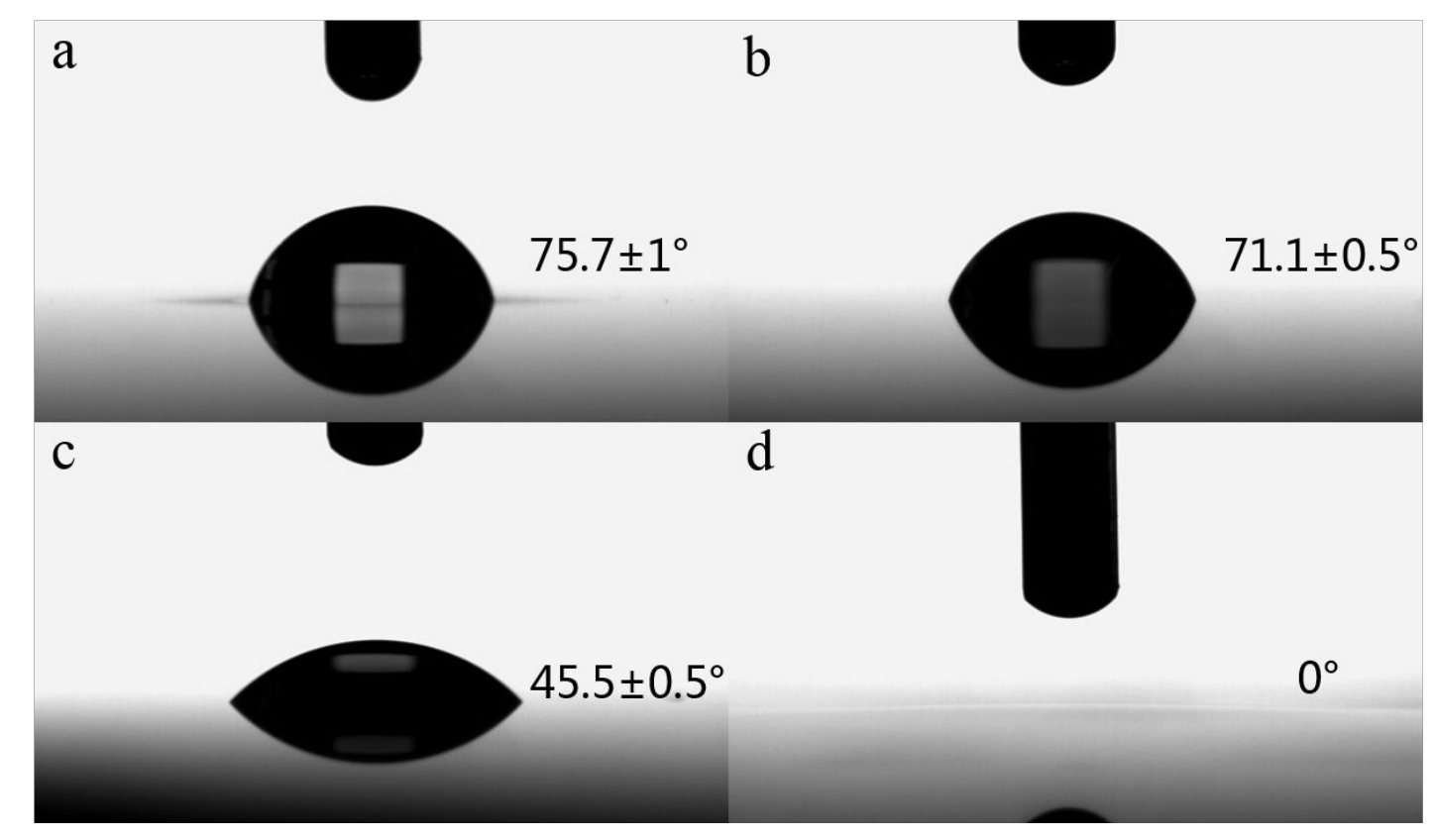

Figure S1. (a-c) Contact angle of DMSO/DMF/IPA onto the POTS-modified wafer surface, illustrating a contact angle of $75.7 \pm 1^{\circ}, 71.1 \pm 0.5^{\circ}$ and $45.5 \pm 0.5^{\circ}$ respectively. (d) Contact angle of the wafer surface exposed to the plasma for $10 \mathrm{~s}$, showing a super-lyophilic state with contact angle of $0^{\circ}$ for the three kinds of solvent. 

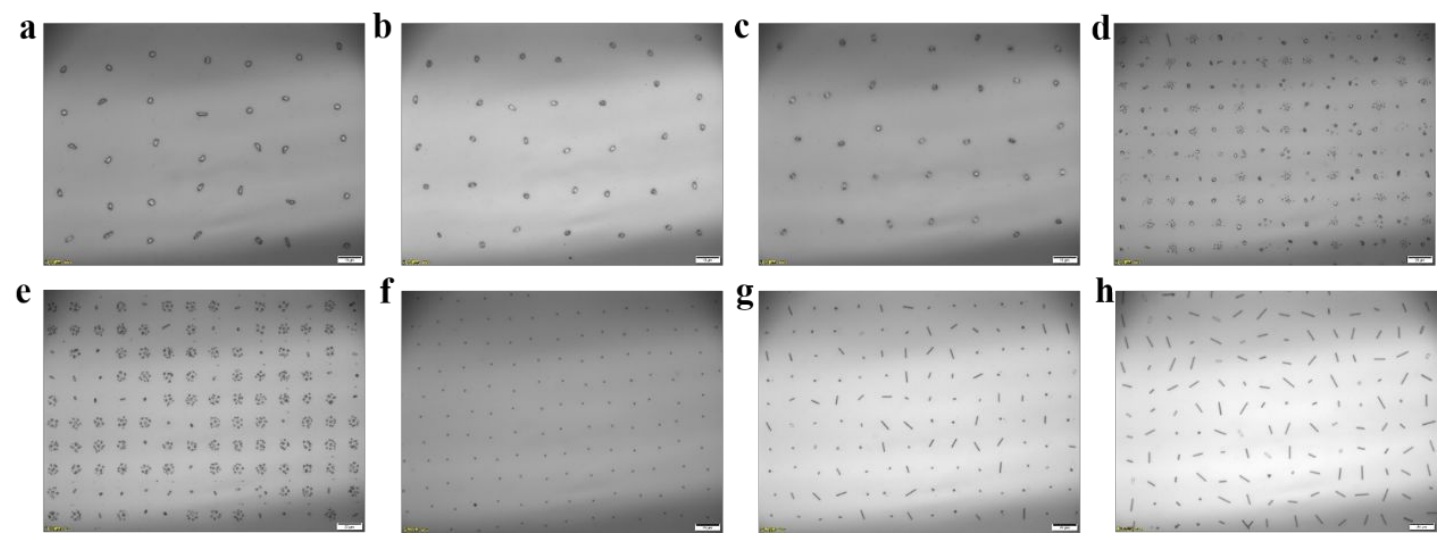

Figure S2. Perovskite crystal array obtained under different conditions including solvent atmosphere, temperature, humidity and precursor concentration. (a-c) Bright field images of crystal array obtained without addition of IPA and DMF atmosphere (a), with addition of DMF atmosphere (b), and addition of IPA atmosphere (c). Scale bar: $10 \mu \mathrm{m}$. (d-e) Bright field images of crystal array obtained under substrate temperature $20{ }^{\circ} \mathrm{C}$ (d), environment humidity $15 \%$ (e), and with precursor concentration of $0.1 \mathrm{M}(\mathrm{f}), 0.2 \mathrm{M}(\mathrm{g}), 0.4 \mathrm{M}(\mathrm{h})$. Scale bar: $20 \mu \mathrm{m}$. 

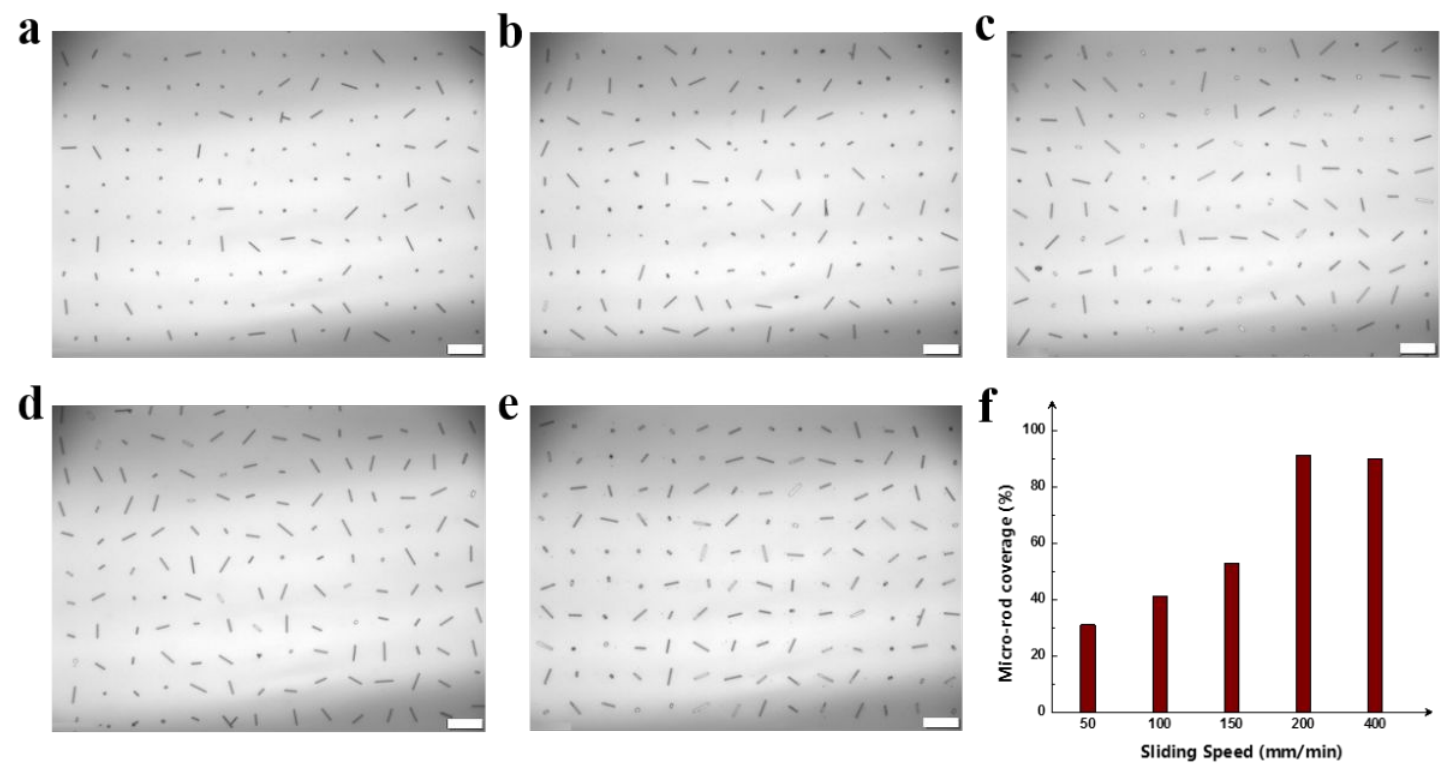

Figure S3. (a-e) Bright field images of perovskite single crystal nanoarray obtained at different sliding speeds ranging from $50 \mathrm{~mm} / \mathrm{min}$ (a), $100 \mathrm{~mm} / \mathrm{min}$ (b), $150 \mathrm{~mm} / \mathrm{min}$ (c), $200 \mathrm{~mm} / \mathrm{min}$ (d) to $400 \mathrm{~mm} / \mathrm{min}$ (e), scale bar: $20 \mu \mathrm{m}$. (f) Histogram of nanorod coverage rate corresponding to different sliding speeds. 

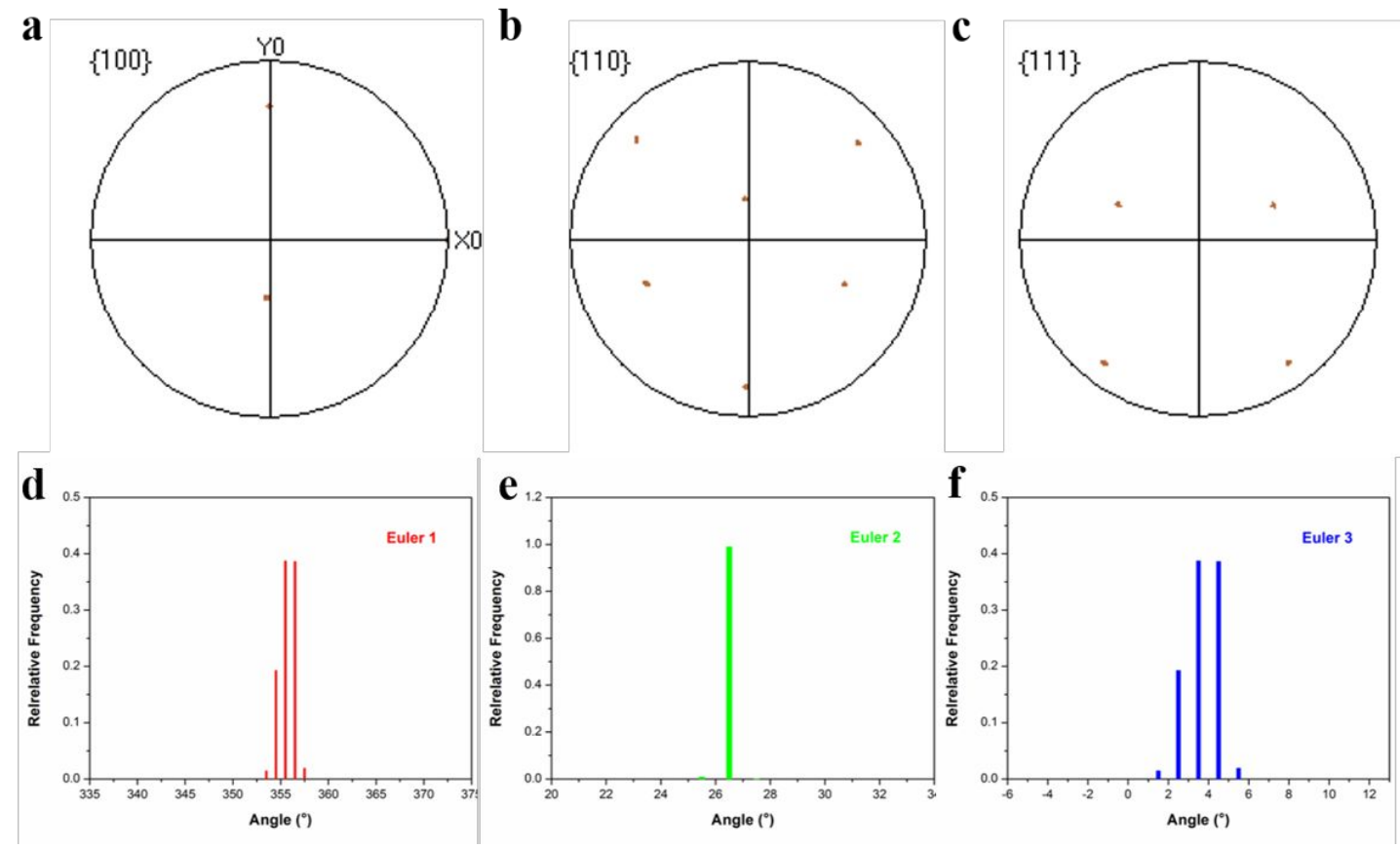

Figure S4. (a-c) The corresponding (100), (110), (111) pole figures for the points. (d-f) Histogram of the distribution of Euler 1, Euler 2, Euler 3 angles. This indicates the single-crystal structure of prepared perovskite crystal further. 


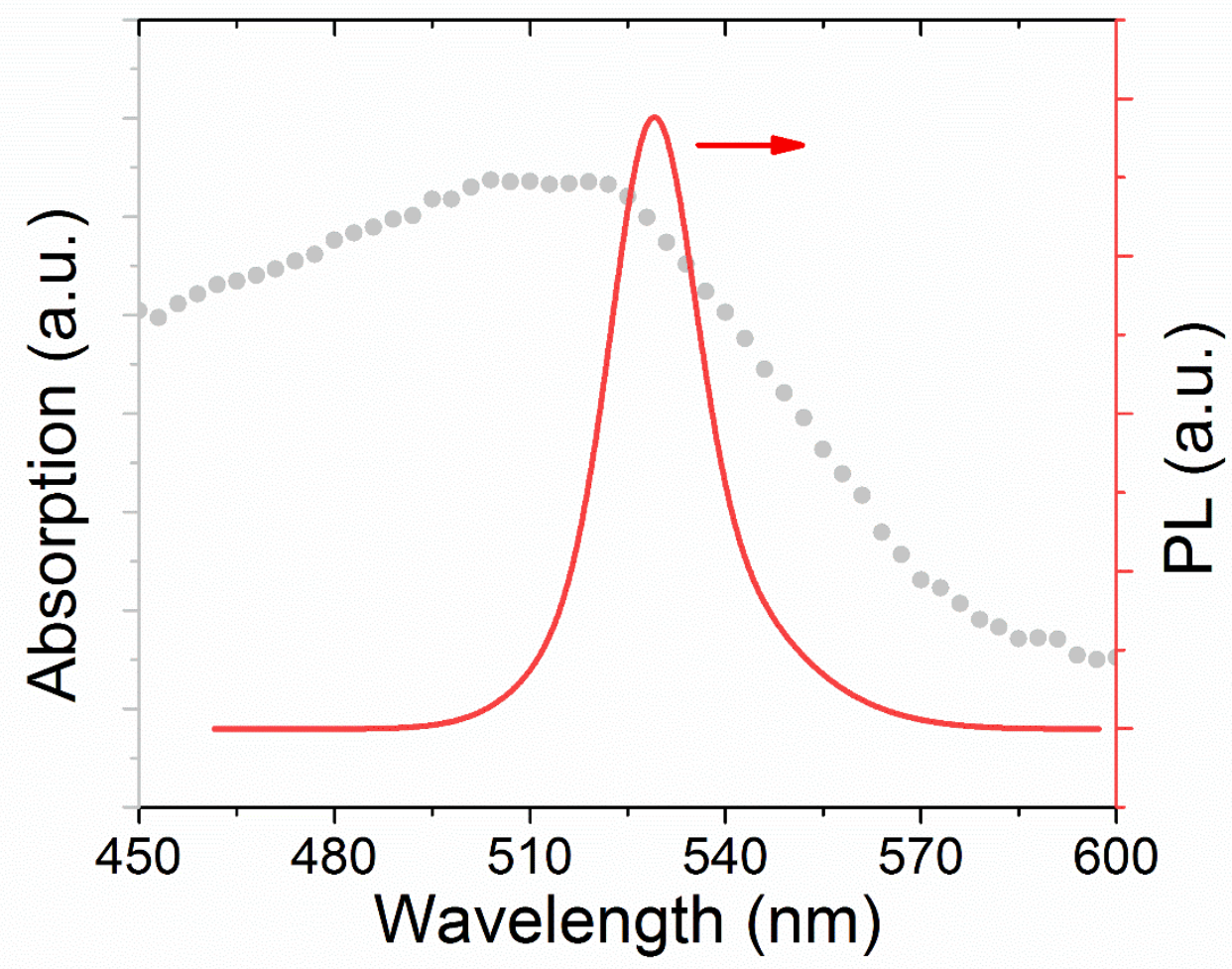

Figure S5. Absorption and $\mathrm{PL}$ spectra of $\mathrm{Cs} \mathrm{PbBr}_{3}$ nanorod array. 

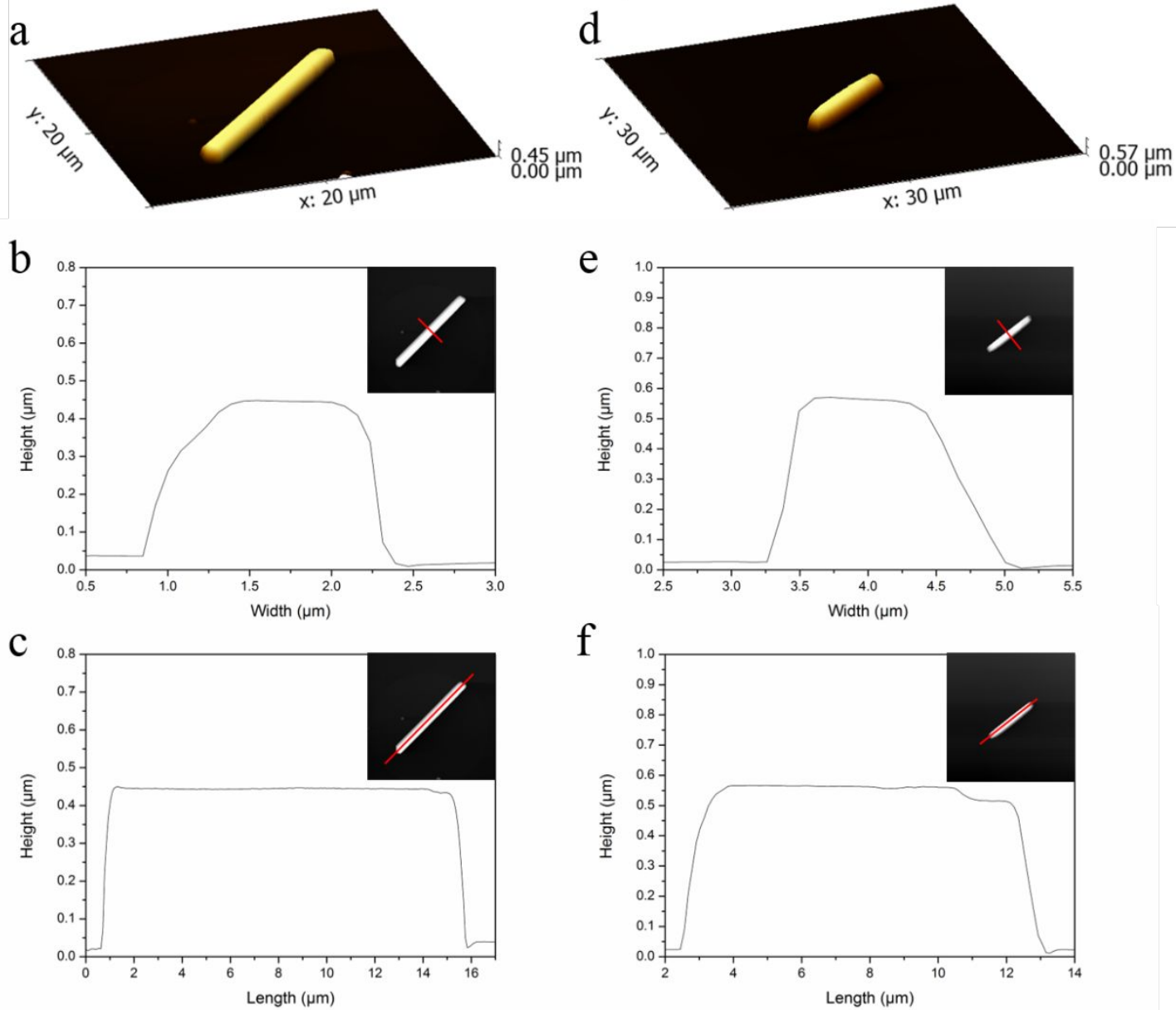

Figure S6. The AFM topography and profiles of $\mathrm{CsPbr}_{3}$ nanorods with different sizes. (a-c) Topography of a $\mathrm{CsPbrr}_{3}$ nanorod with thickness of $422 \mathrm{~nm}$, length of $15.18 \mu \mathrm{m}$ and width of $1.54 \mu \mathrm{m}$. (d-f) Topography of a $\mathrm{CsPbBr} 3$ nanorod with thickness of $545 \mathrm{~nm}$, length of $10.70 \mu \mathrm{m}$ and width of $1.77 \mu \mathrm{m}$. The volume of the two nanorods was calculated to be basically the same, proving the generation mechanism of random crystal size from the side.
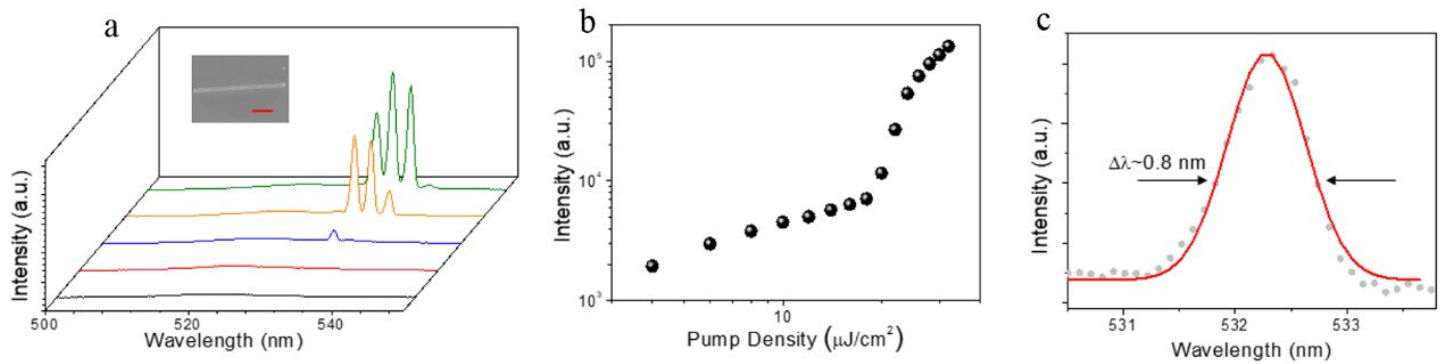
Figure S7. (a) Representative spectra under different pump densities. Inset shows the SEM image of the perovskite nanorod where the scale bar is $2 \mu \mathrm{m}$. (b) The log-log plot of output intensity versus pump density. (c) The high-resolution spectrum collected around the lasing threshold.
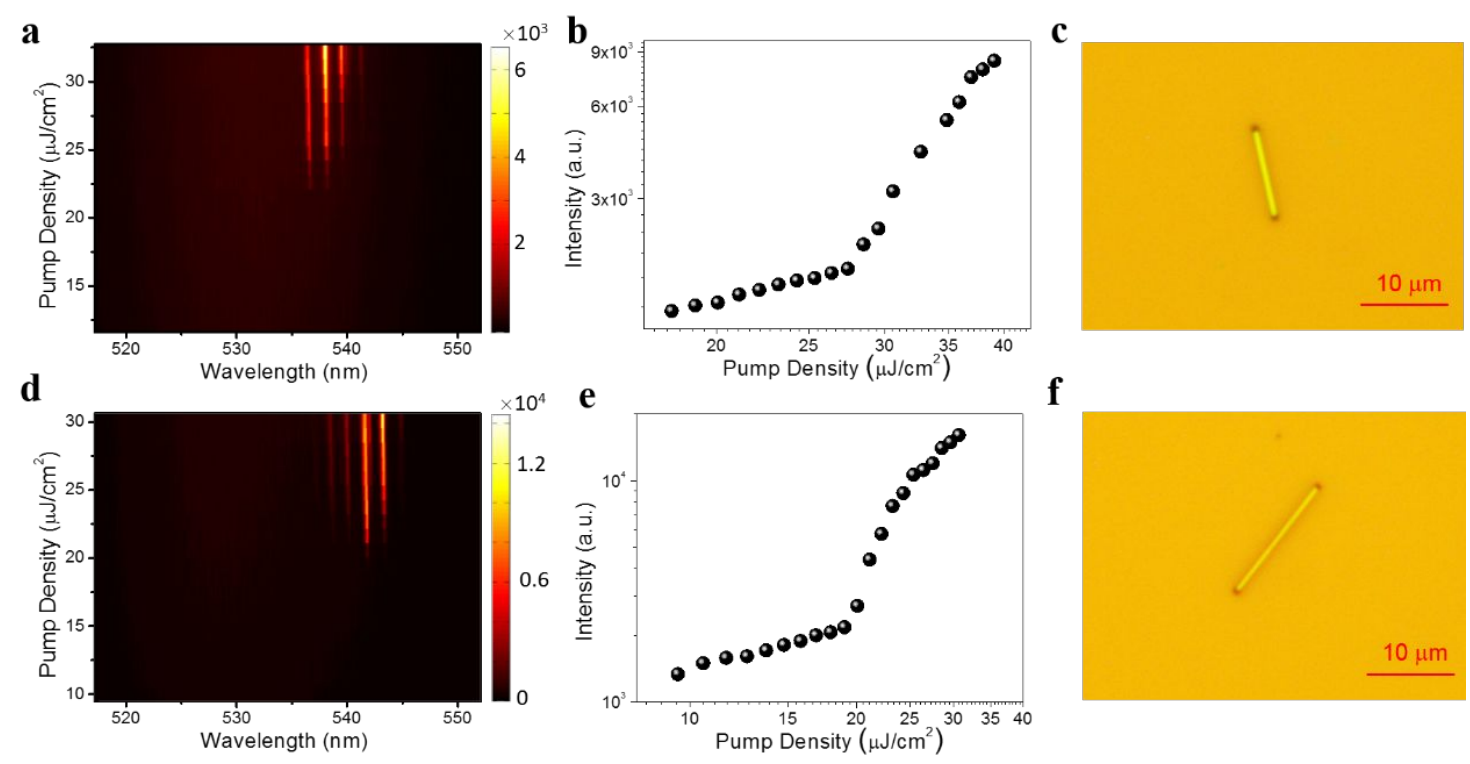

Figure S8. 2D pseudo-color plot of the emission spectrum (a, d) from the corresponding perovskite nanorods (c, f) with the increase of pump density. (b, e) Log-log plot of the integrated emission intensity versus the pump density. 

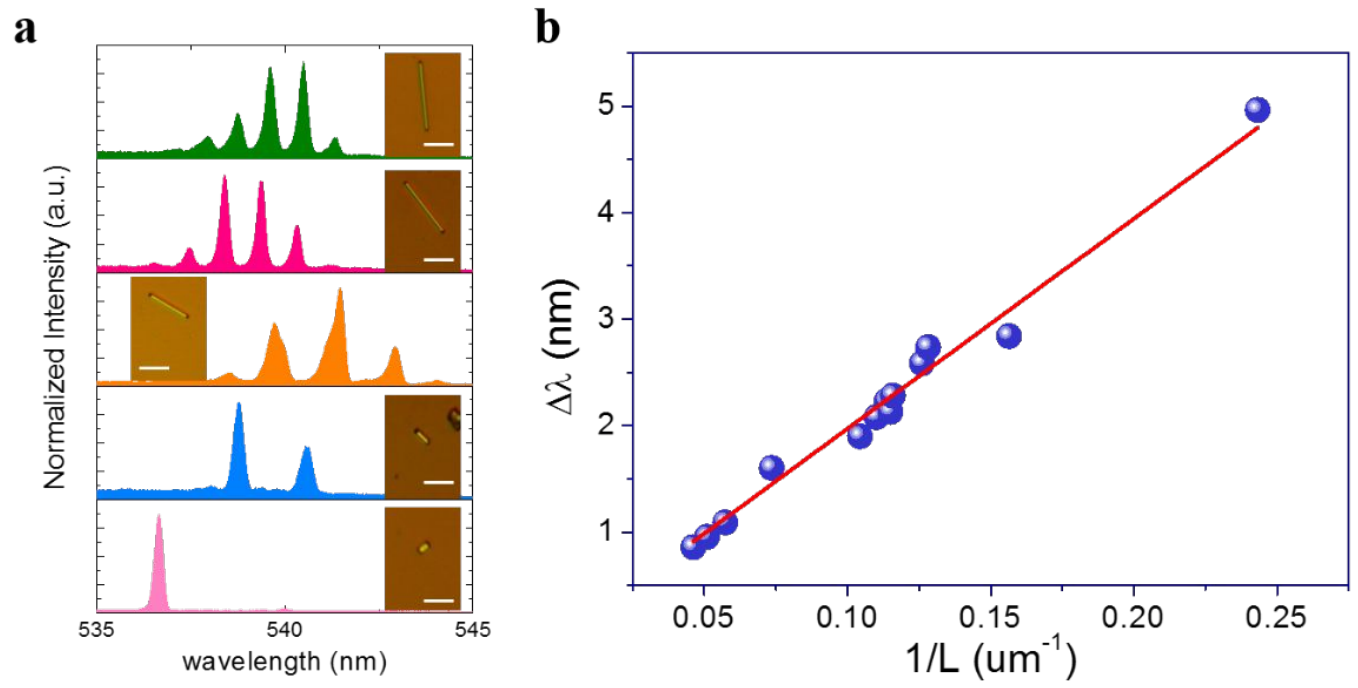

Figure S9. (a) Representative lasing spectra showing the mode spacing evolution with perovskite nanorod length. Insets show the corresponding optical microscopic images with scale bar of $10 \mu \mathrm{m}$. (b) The dependence of mode spacing on the nanorod length. 


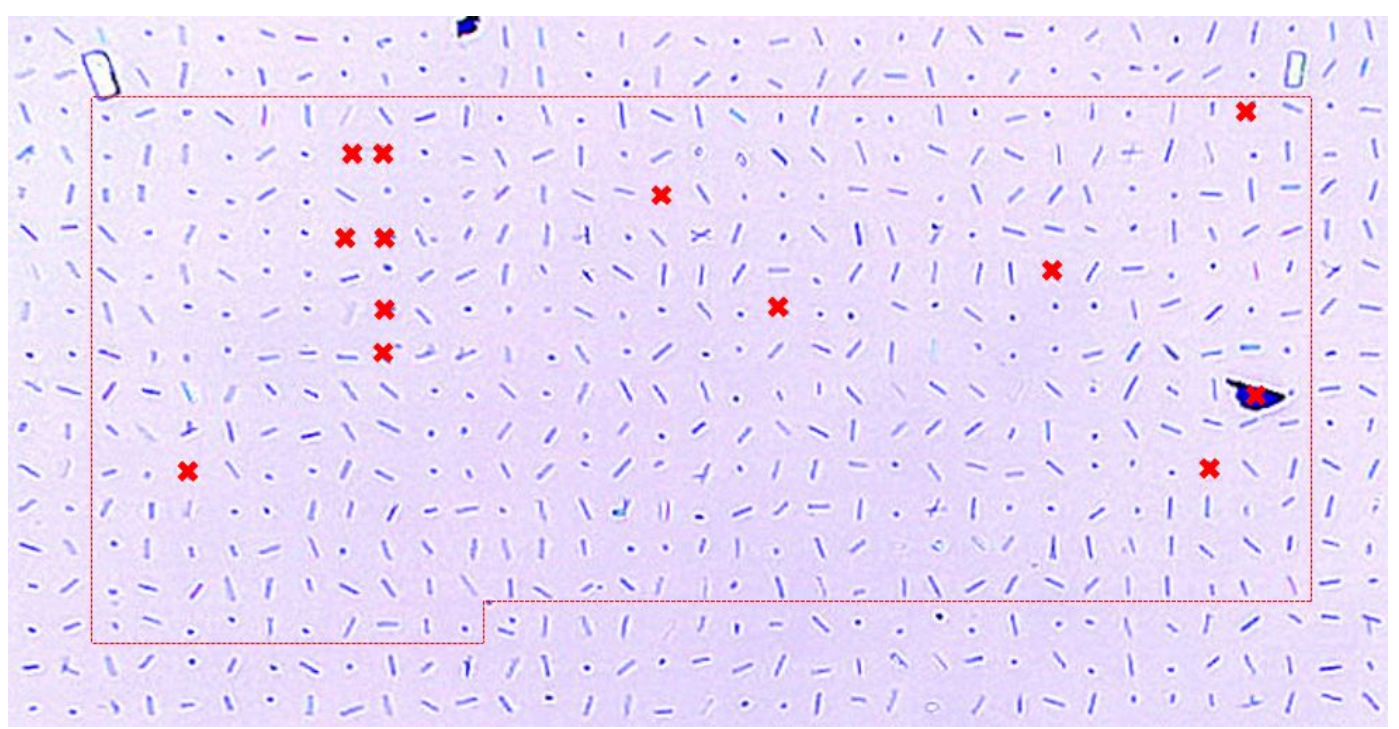

Figure S10. The optical image of the nanorod array marked by the red dashed line. The cross marked the excluded samples. 

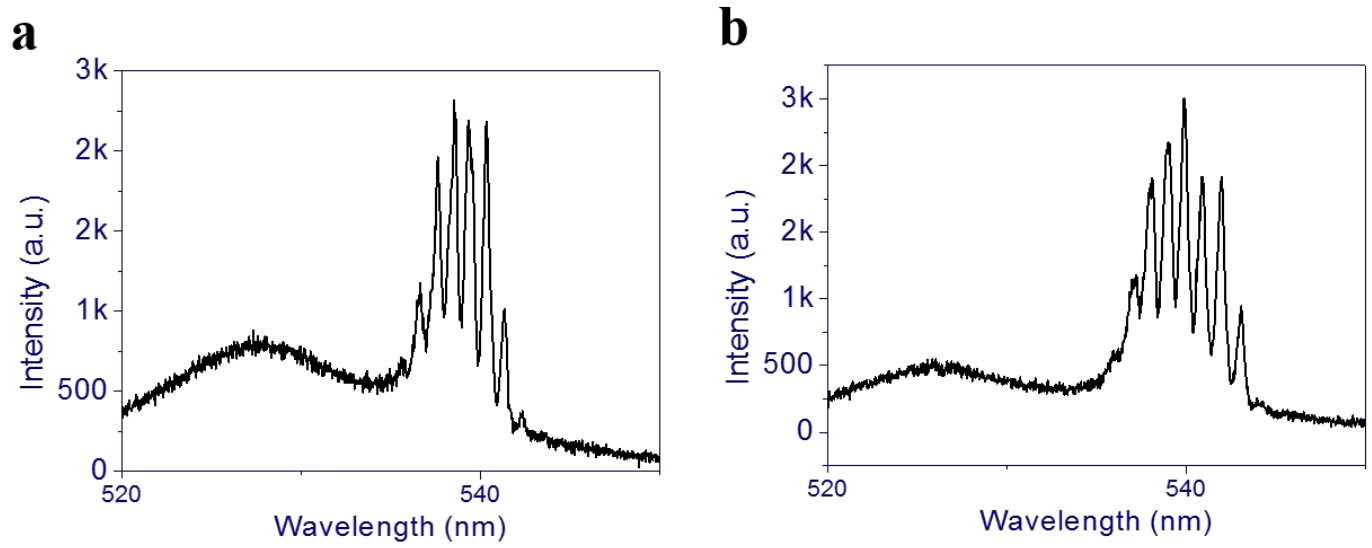

Figure S11. The emission spectra with six (a) and seven (b) lasing modes from the perovskite nanorods. 

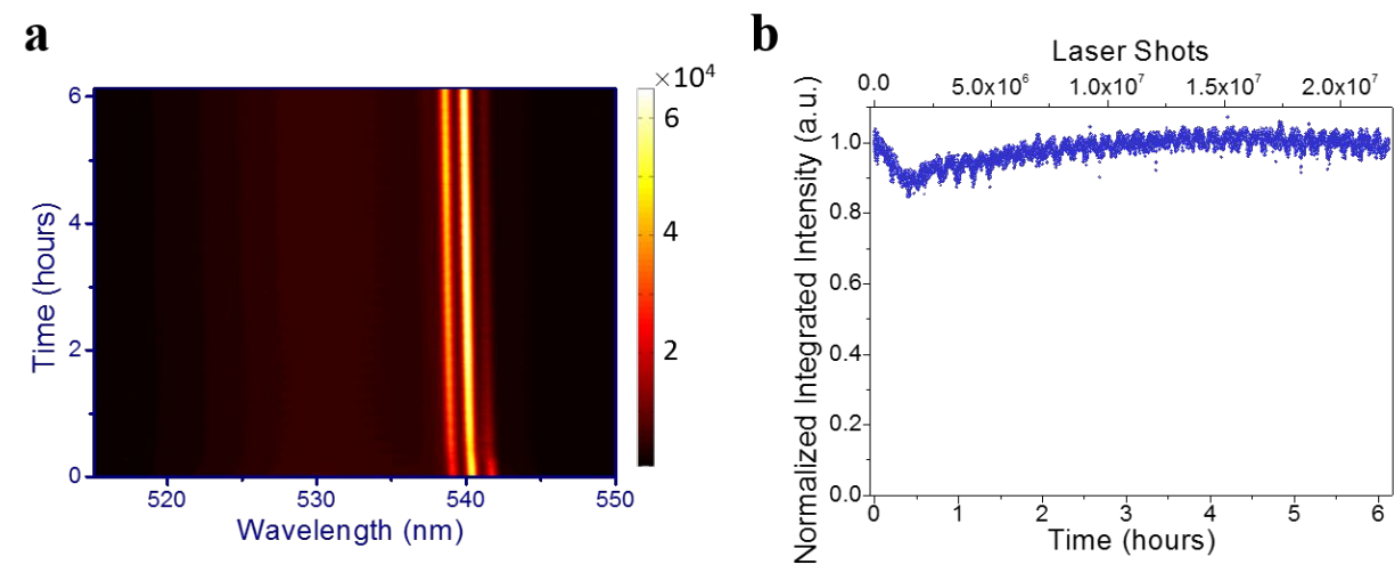

Figure S12. (a) 2D pseudo-color plot of the lasing spectrum evolution with illumination time under a fixed pump fluence in the ambient condition. (b) The integrated intensity as a function of pump time (pump laser shots). 


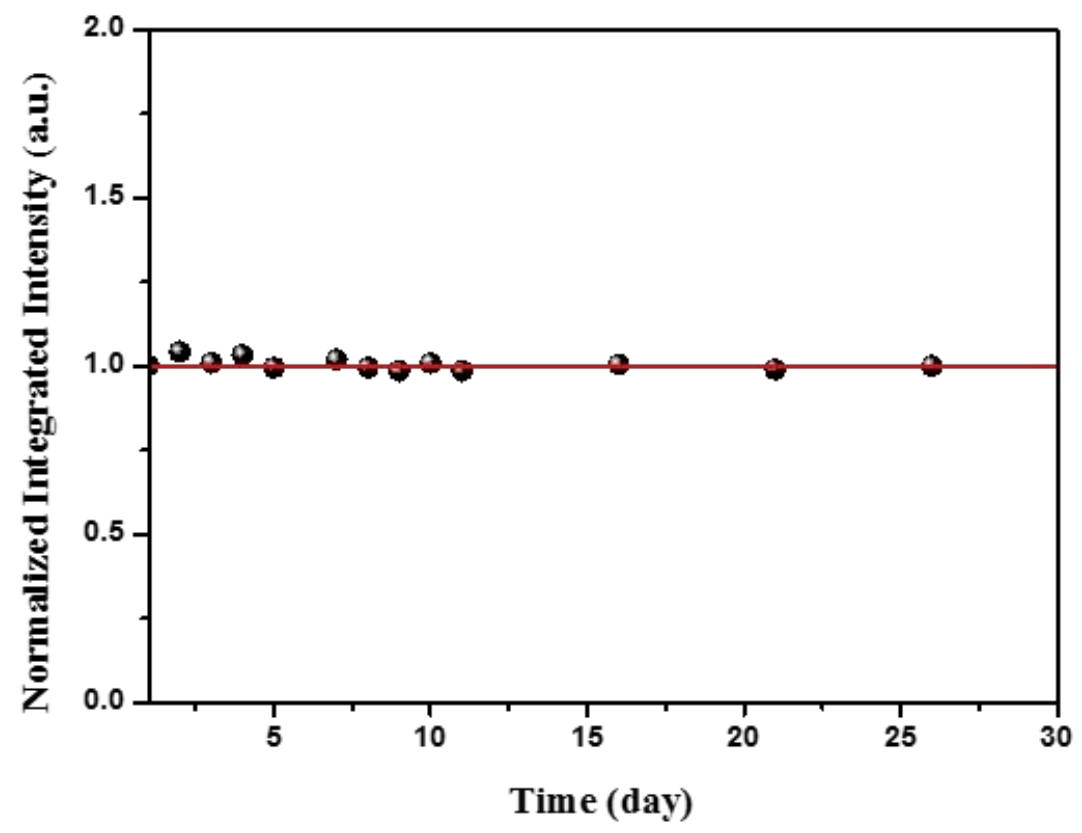

Figure S13. Normalized integrated PL intensity of the perovskite nanorod array under UV light (360-380 nm) irradiation, recorded 30 days. 


\section{Note S1 Calculation of Hamming distance}

In out manuscript, we calculated interclass normalized Hamming distance, which can be defined as,

$$
\mathrm{HD}=\frac{\|S(i) \oplus S(j)\|}{n}
$$

where HD is normalized Hamming distance, S(i) and S(j) represent to two different strings, $\mathrm{n}$ is the number of bits in a string, is the exclusive-or (XOR) operator. For example, two 8-bit strings "10111010" and "10010010" have two different bits. Therefore, the HD is 0.25 in this case. 


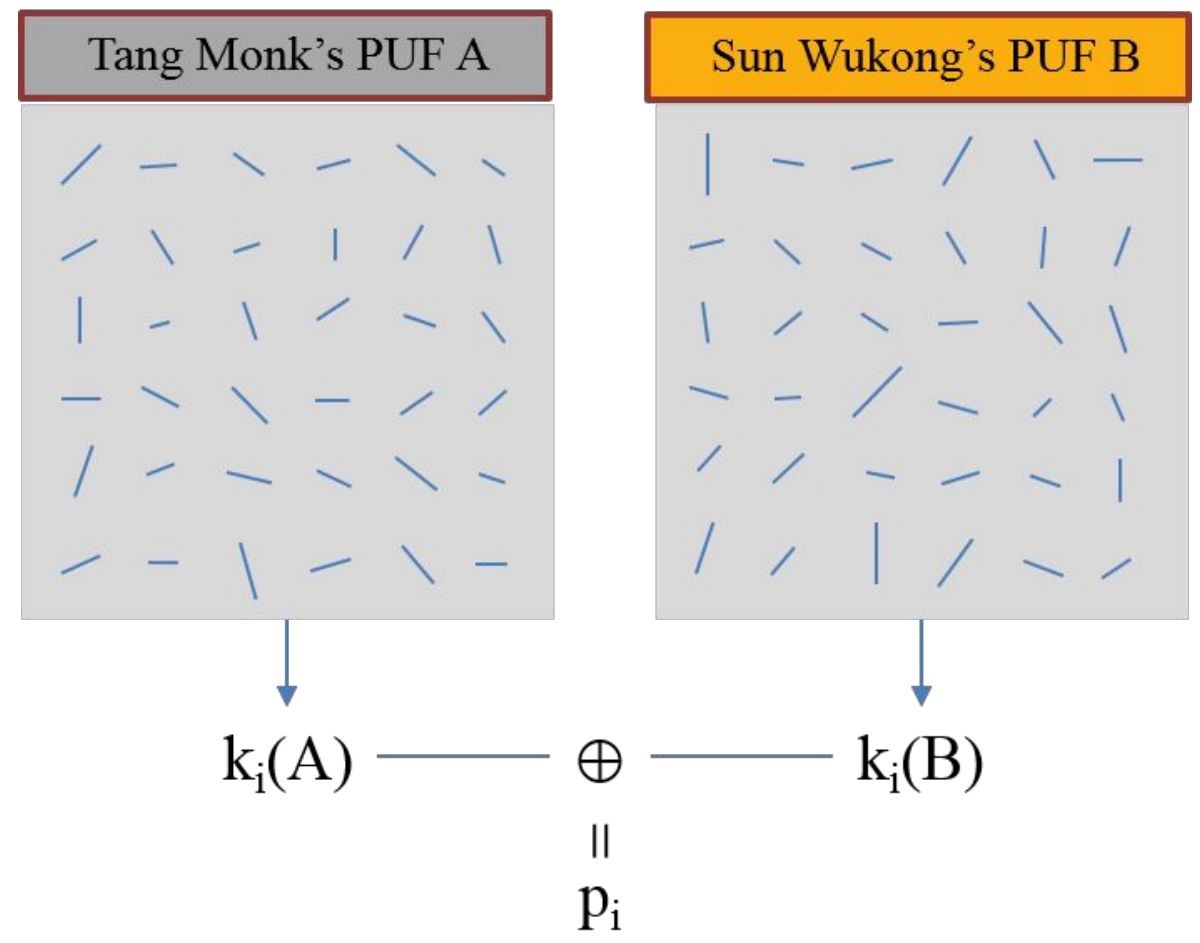

Figure S14. Schematic diagrams of private and public keys generated from single-crystal array chips. 